\title{
Toxina botulínica tipo A nas DTM musculares: há eficácia?
}

\author{
Botulinum toxin type $A$ in muscle TMD: There's \\ effectiveness?
}

\begin{abstract}
RESUMO
Os objetivos dessa revisão da literatura foram verificar a eficácia da toxina botulínica tipo A (BTX-A) na diminuição da dor em indivíduos com DTM e identificar os parâmetros ideais para o local, número de aplicações, dosagens e tempo de duração. Foram selecionados 19 artigos das bases de dados do Google Acadêmico e PubMed, que incluíram 14 artigos de pesquisa clínica e 5 de revisão sistemática. Foi possível concluir a respeito da toxina botulínica que os músculos indicados para a aplicação são principalmente os masseteres e os temporais, podendo ser aplicado também nos músculos pterigoideos, lateral e medial, digástrico e platisma. Os locais de escolha são os que apresentam maior volume e sensibilidade à palpação (pontos-gatilho) ou maior atividade eletromiográfica em repouso. As dosagens variam de um total de $10 \mathrm{U}$ a $400 \mathrm{U}$ de BTX-A por indivíduo, sendo distribuídas pelos músculos indicados. A BTX-A, em geral, é aplicada em dose única, porem alguns autores preconizam uma segunda aplicação se a primeira não fez o efeito esperado. $\mathrm{O}$ efeito da toxina botulínica sobre os músculos e a dor, em geral, tem duração variada, sendo relatado desde 3 a 4 semanas até 3 a 5 meses. A maioria dos estudos observou à eficácia da BTX-A na diminuição da dor de indivíduos com DTM. Porém é necessário que mais estudos clínicos randomizados, duplo cegos, multicêntricos e controlados sejam realizados para que a eficácia da BTX-A seja comprovada e para que um protocolo de atendimento seja realizado.

Palavras-chave: Síndrome da Dor Miofascial; Toxina Botulínica Tipo A; Transtornos da Articulação Temporomandibular.
\end{abstract}

\footnotetext{
ABSTRACT

The objectives of this literature review were to verify the efficacy of botulinum toxin type A (BTX-A) in reducing pain of TMD patients and to identify the optimal parameters for the location, number of applications, dosages and duration. We selected 19 articles from Google Scholar and PubMed databases that included 14 articles of clinical research and 5 systematic reviews. It was concluded about BTX-A that the muscles appropriate to the application are mostly masseter and temporal and can also be applied in the pterygoid muscle lateral and medial, digastric and platysma. The choices of locations are those who have higher volume and sensitivity to palpation (trigger points) or higher EMG activity at rest. Dosages vary from a total of $10 \mathrm{U}$ to $400 \mathrm{U}$ of BTX-A by individual, being distributed by the indicated muscles. BTX-A in general is applied in a single dose, but some authors recommend a second application if the first did not make the expected effect. The effect of BTX-A on muscle and pain in general has varying duration, being reported from 3 to 4 weeks for 3 to 5 months. Most studies have noted at the effectiveness of BTX-A in patient pain reduction DTM. However more randomized, double-blind, multicenter, controlled clinical trials needs to be carried out so that the effectiveness of BTX-A could be confirmed and a management protocol, stabilished.

Key words: Myofascial Pain Syndromes; Botulinum Toxins Type A; Temporomandibular Joint Disorders
}

\section{Melina Triana Fassina*}

Renato Morales Jóias**

Renata Pilli Jóias***

* DDS, Curso de Odontologia, Faculdade de Ciências Médicas e da Saúde, Universidade

Metodista de São Paulo

** DDS, MsC, PhD, Professor Assitente

do Curso de Odontologia, Faculdade de

Ciências Médicas e da Saúde, Universidade

Metodista de São Paulo

*** DDS, MsC, PhD, Professora Assitente do

Curso de Odontologia e Coordenadora do

Curso de de Especialização em Ortodontia,

Faculdade de Ciências Médicas e da Saúde,

Universidade Metodista de São Paulo

Endereço para correspondência

Melina Triana Fassina*

Rua Cedral, 157 - Vila Progresso, Santo

André, SP

Telefone: (11) 4458-1557

Celular: (11) 98424-5749

Email: melinafassina@gmail.com

Submetido em: 11-9-2016

Aceito em: 26-7-2017 


\section{INTRODUÇÃO}

As desordens temporomandibulares (DTM) compreendem um grupo de condições de dor crônica que afeta os músculos mastigatórios e as articulações temporomandibulare $^{1-2}$ com restrição no movimento mandibular e presença de sons articulares durante o movimento, podendo ser classificadas como muscular, articular ou mista ${ }^{3}$. A DTM muscular é o mais comum, sendo muitas vezes mal localizada e referida ao pescoço, face, dentes ou regiões pré-auricular e, por vezes, acompanhada de dores de cabeça e limitação dos movimentos mandibulares ${ }^{2}$.

Diversos tipos de tratamento para DTM muscular são sugeridos na literatura como terapia medicamentosa, acupuntura, fisioterapia, tratamento psicológico, entre outros ${ }^{4-5-6}$. A terapia com placa oclusal é um método universal entre os dentistas além de ser extremamente eficaz, possibilitando a diminuição da sintomatologia dolorosa em até 90\% e a melhora da qualidade de vida ${ }^{4,6-7-8}$

Embora as placas apresentem grande eficácia para o controle da dor e de grande utilização, é necessário que o operador seja capaz de realizar ajustes, tornando o procedimento trabalhoso. É necessária a busca constante por métodos que promovam mais conforto para o paciente e maior praticidade nas etapas clínicas. Uma solução que está sendo estudada e mostrando eficácia é o tratamento com a toxina botulínica que, se trouxer benefício ao paciente, é uma forma mais prática por ser de única aplicação.

Produzida pelo Clostridium Botulinum, a toxina botulínica consiste de uma mistura complexa de proteínas e pode ser dividida em sete sorotipos, sendo o tipo A (BTX-A) o mais amplamente estudado para fins terapêuticos ${ }^{9-10}$. Ela provoca relaxamento muscular, bloqueando temporariamente a liberação de acetilcolina nos terminais nervosos colinérgicos pré-sinápticos e o músculo permanece paralisado enquanto estiver sob seu efeito².

Um grupo diversificado de DTM (bruxismo e apertamento; dor miofascial; dor miofascial com o envolvimento da ATM secundário; trismo; hipertrofia dos músculos masseter e temporal; e cefaleias) tem mostrado evidência inicial de ser passível ao tratamento com a toxina botulínica ${ }^{11}$. Ela vem emergindo como uma ferramenta clínica em potencial e valiosa para o diagnóstico e a terapêutica de DTM, obrigando-nos a reexaminar a nossa compreensão sobre as DTM e suas origens ${ }^{11}$.

Os estudos ainda são controversos sobre o real beneficio da toxina botulínica e por isso há a necessidade de entender melhor os locais, as dosagens, o número de aplicação, o tempo de duração e verificar a eficácia na diminuição da dor em indivíduos com DTM. Por isso a proposta de uma revisão da literatura a esse respeito, para identificar os parâmetros ideais para um protocolo de aplicação.

\section{REVISÃO DE LITERATURA}

Em 1994, foi realizado um estudo preliminar controlado por placebo, randomizado, duplo-cego para demonstrar o sucesso obtido com injeções de toxina botulínica. Seis pacientes com dor miofascial crônica foram selecionados para receber injeções de toxina botulínica tipo A (BTX-A) ou solução salina nos pontos gatilho. A dor foi medida pela escala analógica visual (EVA), descritores verbais para a intensidade da dor e desconforto, 
firmeza muscular palpável e limiares de dor à pressão (algômetro). Os pacientes foram divididos em grupos que receberam injeções de $50 \mathrm{U}$ de BTX-A ou de $4 \mathrm{ml}$ de solução salina em dois tempos com 8 semanas de intervalo e foram acompanhados em intervalos semanais durante 4 semanas após cada injeção e 8 semanas após a injeção final. Quatro pacientes apresentaram redução da dor de pelo menos 30\%, após aplicação de BTX-A, mas não nas injeções de solução salina. Os resultados foram estatisticamente significativo de 2 a 4 semanas para o EVA, intensidade da dor (exceto a 2 semanas), espasmo e sensibilidade, mas não para desagrado da dor. Os pacientes relataram que a melhora após a última injeção durou de 3 a 4 semanas, tanto para BTX-A quanto para o placebo. Os autores concluíram que o tratamento com a BTX-A mostrou-se eficaz para dor miofascial, mas que estes resultados devem ser confirmados por um estudo mais amplo ${ }^{12}$.

Já em 1999, um estudo não controlado foi realizado com o objetivo de avaliar a utilização da BTX-A em pacientes com DTM. Quinze indivíduos foram selecionados e tratados com injeções de 150U de BTX-A, sendo 50U em cada músculo masseter e 25U em cada músculo temporal sob orientação eletromiográfica (EMG). A avaliação foi feita em intervalos de 2 semanas, durante 8 semanas. As medidas avaliadas incluíram dor subjetiva pela escala analógica visual (EVA), força de mordida, abertura da boca, sensibilidade à palpação e um índice funcional com base no EVA. Todas as medidas, com exceção da força de mordida, mostraram uma diferença significativa entre a avaliação de pré-tratamento e as quatro avaliações seguintes. Os autores concluíram que, em quatro dos cinco resultados medidos apresentaram melhoras significativas após as injeções de BTX- $\mathrm{A}^{13}$.

Uma pesquisa com 46 pacientes diagnosticados com DTM miofascial, ou DTM miofacial com desarranjos articulares internos ou artralgia, ou DTM miofascial com desarranjos articulares internos e artralgia, foi realizada com o objetivo de avaliar as respostas subjetiva e objetiva da BTX-A para o tratamento de DTM. Os pacientes foram tratados com 150U de BTX-A, sendo 50U em cada músculo masseter e 25U nos músculos temporais, de acordo com a área de maior massa muscular à palpação e maior atividade no EMG. Em cada aplicação, foi avaliada a dor de forma subjetiva pelo EVA e a contração máxima voluntária (CMV), por meio da EMG dos músculos masseter e temporal, abertura de boca, sensibilidade à palpação e um índice funcional baseado em EVA. A avaliação ocorreu a cada duas semanas, durante oito semanas. Os resultados entre a avaliação pré-tratamento e as quatro avaliações de acompanhamento demonstraram diferenças significativas. A CMV reduziu em 2 meses, mas retornou aos valores do pré-tratamento nas duas avaliações finais. As outras medidas permaneceram significativamente diferentes dos valores do pré-tratamento. Embora o estudo não tenha sido controlado, os autores obtiveram resultados significativos nas injeções de BTX-A ${ }^{1}$.

Com o objetivo de avaliar o potencial terapêutico da BTX-A no tratamento da hiperatividade dolorosa dos músculos mastigatórios, um estudo com 40 pacientes com DTM muscular foi realizado. Foram injetados 200U BTX-A nos músculos mastigatórios com maior atividade eletromiográfica em repouso. Em 8 casos, a injeção intramuscular de BTX-A foi administrada nos músculos pterigóideo lateral, músculo digástrico e músculo pterigoideo medial, as demais injeções foram administradas nos músculos masseter, temporal e platisma. Os pacientes foram observados durante 3 a 12 meses. Os resultados mostraram que $80 \%$ dos pacientes obtiveram melhoras por uma redução média de $45 \%$ 
sobre EVA. Durante o período de observação, 17\% dos pacientes tiveram que receber uma segunda injeção devido à dor recorrente. Segundo os autores, embora os resultados sejam encorajadores, é necessário serem confirmados por estudos randomizados controlados ${ }^{14}$.

Com base nos estudos realizados sobre o sucesso da toxina botulínica para a DTM, foi realizada uma pesquisa para descrever os tipos mais prevalentes (bruxismo e apertamento; distonias oromandibular; dor miofascial; dor miofascial com o envolvimento da ATM secundário; trismo; hipertrofia dos músculos masseter e temporal; e dores de cabeça) e o tratamento com a BTX-A, a dosagem e os locais das injeções de cada músculo. No músculo temporal: 5 injeções de 5 a 25U BTX-A; músculo masseter: 5 injeções de 25 a $50 \mathrm{U}$ BTX-A nas áreas de maior atividade eletromiográfica (EMG), com maior massa e/ou maior desconforto; músculos pterigoide medial: 2 a 3 injeções de 5 a 25U de BTX-A podendo ser injetado via submandibular no músculo extraoral ou através de uma palpação na via intraoral; músculo pterigoide lateral: 1 injeção de 5 a 10U BTX-A com orientação EMG, podendo ser intraoral ou extraoral. A dose é individualizada para o paciente e depende do tamanho do músculo, da dor e da atividade EMG. Os autores afirmam que a toxina botulínica está emergindo como uma ferramenta clínica muito potente e valiosa para o cuidado de diagnóstico e de terapêutica de DTM, mas ensaios clínicos são necessários para comprovar sua eficácia ${ }^{11}$.

Foi realizado um estudo prospectivo, cego, randomizado, controlado por placebo com o objetivo de avaliar se o tratamento com injeção de BTX-A na redução da hiperatividade muscular mastigatória pode melhorar os sintomas faciais de dor de cabeça no caso de outros métodos de tratamento ter sido ineficazes. Noventa indivíduos com dor facial crônica participaram do estudo, sendo que 30 receberam tratamento placebo, os demais foram tratados com injeções de 35U de BTX-A nos músculos masseter, temporal e pterigóideo medial. Noventa e um por cento dos pacientes que receberam BTX-A demonstraram melhora de aproximadamente 3.2 no EVA, indicando uma diferença significante em comparação com o grupo placebo. Os autores concluíram que a injeção local de BTX-A é um método inovador e eficiente para o tratamento da dor facial crônica associada com hiperatividade dos músculos mastigatórios, sendo uma opção para os pacientes que não respondem a métodos de tratamento conservador ${ }^{15}$.

A BTX-A é utilizada para tratar várias condições de dor, incluindo espasticidade muscular, distonia, dor de cabeça e dor miofascial. A literatura é conflitante com relação ao uso de BTX-A para injeção no ponto gatilho para melhora de dor, por tanto o objetivo do estudo foi realizar uma revisão sistemática para avaliar a evidência da eficácia da BTX-A em comparação com outros tratamentos (anestésico local, corticosteroides, injeção de solução salina e agulhamento seco) e placebo. A Escala de Dor Válida de Oxford foi utilizada como critério de seleção e classificação dos ensaios clínicos, no qual 5 itens com pontuação de 0-16 foram avaliados por meio da Escala de Avaliação Oxford. Dados extraídos de ensaios qualificados incluíram medidas de resultados, como a intensidade da dor e limiar de pressão dor e as conclusões dos autores foram comparadas. De 21 ensaios clínicos relevantes, cinco preencheram os critérios de inclusão. Um estudo concluiu que BTX-A foi eficaz, e 4 concluíram que não era eficaz para reduzir a dor decorrente de pontos-gatilho. Os autores não apoiaram a utilização de BTX-A na redução da dor associada com os pontos-gatilho, pois as evidências não suportam sua eficácia ${ }^{16}$. 
Um ensaio clínico randomizado, duplo-cego, controlado com grupo placebo foi realizado para estudar o efeito da BTX-A sobre o ponto-gatilho dos músculos doloridos e com o objetivo de esclarecer o seu possível efeito analgésico. Foram incluídos no estudo 30 pacientes com pontos de desencadeamento de dor no músculo infraespinoso, que receberam 50U de BTX-A e 0,25 mL de solução salina. Após 3 e 28 dias da aplicação, foram avaliados os seguintes parâmetros: dor local e referida espontânea, detecção de dor pelo EVA, limiar de tolerância à pressão mecânica e o movimento do ombro. Os resultados indicaram que houve uma redução significativa na atividade da placa motora e no padrão de interferência no EMG após o uso do BTX-A, mas não teve efeito sobre qualquer dor (espontânea ou referenciada) ou o limiar da dor em comparação com solução salina. Com isso, os resultados não suportaram um efeito antinociceptivo e analgésico da BTX-A e mais estudos precisam ser realizados ${ }^{17}$.

Foi realizado um estudo prospectivo, randomizado, duplo-cego, multicêntrico, controlado por placebo de 12 semanas para avaliar a eficácia e a tolerabilidade da BTX-A em pacientes com síndrome dolorosa miofascial que afetavam músculos da parte superior das costas. Foram incluídos no estudo pacientes que tivessem pelo menos 10 pontos-gatilho e uma duração da doença de 6-24 meses. Cento e quarenta e cinco pacientes foram randomizados para receber injeções de BTX-A ou injeções de solução salina, que foram feitas nos 10 pontos gatilho mais sensíveis (40U por local). O desfecho primário foi a proporção de pacientes com dor leve ou nenhuma dor na semana 5 e o desfecho secundário incluiu mudança na intensidade da dor, duração da dor, número de dias livres da dor por semana, duração do sono, número e intensidade da dor nos pontos-gatilho e o tempo para melhora na dor. Na semana 5, mais pacientes no grupo BTX-A relataram significativa melhora ou ausência de dor, em comparação com os pacientes no grupo placebo. O grupo que recebeu injeções de BTX-A apresentou maior diminuição da intensidade da dor durante as semanas 5-8, e mais dias por semana sem dor entre 5 e 12 semanas. Com isso, o uso da BTX-A em pacientes com síndrome dolorosa miofascial na parte superior das costas mostram um resultado positivo na dor ${ }^{18}$.

Com o objetivo avaliar a eficácia da BTX-A pro tratamento dos sintomas de dor miofascial e pra redução da hiperatividade muscular em indivíduos com bruxismo, foi realizado um estudo preliminar randomizado, duplo-cego, controlado por placebo. Vinte pacientes diagnosticados com bruxismo e dor miofascial foram selecionados e divididos aleatoriamente em dois grupos. Um grupo recebeu injeções num total de 100U de BTX-A em uma sessão única, sendo 30U em cada músculo masseter e 20U em cada músculo temporal, e um grupo controle recebeu injeções de placebo. Parâmetros clínicos objetivos e subjetivos como: dor em repouso e durante a mastigação, eficiência mastigatória, movimentos máximos de abertura da boca não assistidos e assistidos, protrusão e laterotrusão, limitação funcional durante movimentos mandibulares habituais, eficácia subjetiva do tratamento e tolerância ao tratamento, foram avaliadas no início do tratamento e após uma semana, um mês, seis meses e em consultas de acompanhamento. O estudo mostrou melhora objetiva (amplitude dos movimentos mandibulares) e subjetiva (dor em repouso e durante a mastigação), sendo uma melhora significativamente maior nos resultados do grupo tratado com BTX-A do que no grupo tratado com placebo, principalmente na dor subjetiva. As diferenças não foram significativas em alguns casos devido ao pequeno ta- 
manho da amostra, porem eles apoiam a eficácia da BTX-A para reduzir os sintomas de dor miofascial em indivíduos com bruxismo ${ }^{19}$.

Devido a poucas pesquisas que analisam injeções nos pontos-gatilho com soluções diferentes, um estudo foi realizado para avaliar a eficácia de injeções nos pontos-gatilho usando BTX, lidocaína e agulhamento seco na diminuição da dor local e no gerenciamento de dor de cabeça associada. Quarenta e cinco pacientes com dor miofascial e dores de cabeça foram distribuídos aleatoriamente em três grupos: G1 com agulhas secas (grupo controle), G2 com 0,25\% de lidocaína a 0,25\% e G3 com 25 a 50U de BTX. Os pacientes foram avaliados durante 12 semanas, sendo observados os níveis de intensidade da dor, frequência e duração, sensibilidade no local após a injeção nos pontos-gatilho, tempo de obtenção e duração do alívio e a utilização de medicação de resgate. Todos os grupos apresentaram resultados favoráveis. Apenas o uso de medicação de resgate e sensibilidade no local após a injeção que o G3 apresentou melhores resultados. Foi concluído que as substâncias testadas têm efeitos desejáveis sobre os distúrbios estudados, porém a lidocaína, pelo baixo custo, poderia ser adotada como a substância de escolha e a BTX deveria ser reservada para casos refratários que não atingiram os efeitos esperados ${ }^{20}$.

Uma pesquisa foi realizada para investigar a eficácia da BTX-A em pacientes com DTM muscular crônica. Participaram 21 indivíduos com DTM muscular que não obtiveram alívio da dor após o tratamento convencional. Cada indivíduo recebeu 50U de BTX-A e solução salina isotônica nos músculos masseter com sintomatologia dolorosa, mas a ordem de injeções era desconhecida para eles e para o avaliador. Os pacientes foram acompanhados por 3 meses, sendo avaliados após 1 e 3 meses. O principal parâmetro avaliado foi a remissão da dor em repouso (por meio do EVA), além disso, foi observada a função física e emocional, melhora global e as medidas adicionais de desfechos, que foram o uso de analgésicos, a capacidade livre de dor na mandíbula na abertura, dor a palpação dos músculos mastigatórios, limiar de dor à pressão e tolerância. Não houve diferença significante na redução da dor com toxina botulínica e solução salina, porém a toxina foi mais rápida. A BTX-A reduziu em média 30 pontos na EVA no primeiro mês e 23 pontos após 3 meses; e a solução salina reduziu 11 pontos e 4, respectivamente. O número de pacientes em que foi observada a redução da dor superior ou igual a 30\% não foi estatisticamente maior para a BTX-A do que após a solução salina em qualquer visita de acompanhamento. Não houve mudanças estatisticamente significantes no pós-tratamento em quaisquer outras medidas de resultado, com exceção de dor à palpação, que diminuiu 3 meses após a injeção de solução salina. Os autores concluíram que a BTX-A não é eficaz como um complemento ao tratamento conservador em pacientes com dor miofascial persistente ${ }^{2}$.

Um estudo controlado randomizado foi realizado para comparar a eficácia a curto prazo de injeções de BTX-A e tratamento por meio de técnicas de manipulação facial na diminuição da dor miofascial. Foram selecionados 30 pacientes com dor miofascial e divididos aleatoriamente em dois grupos. Grupo A recebeu injeções de 150U de BTX-A nos músculos masseter e temporal em uma sessão única. Grupo B, 3 sessões de 50 minutos (uma por semana) de manipulação facial. Níveis de dor máxima (classificação EVA) e gama de movimento da mandíbula em milímetros (máxima abertura de boca, protrusão direita e esquerda e laterotrusão) foram avaliados no início, no final e após 3 meses do tratamento. Em ambos os protocolos de tratamento foram observada melhora significativa ao longo do 
tempo para os sintomas da dor. Os dois tratamentos demonstraram-se quase igualmente eficazes, sendo a manipulação facial ligeiramente superior na redução da percepção da dor subjetiva (EVA), e as injeções de BTX-A sendo ligeiramente superior no aumento da amplitude de movimento da mandíbula. As diferenças entre os dois protocolos de tratamento não foram relevantes clinicamente. Os autores acreditam que estudos futuros com amostras maiores e acompanhamento longitudinal são necessários a fim de elucidar as melhores estratégias de tratamento para este quadro clínico ${ }^{10}$.

Apesar da ampla prevalência de distúrbios da dor miofascial e por ser o primeiro grupo de doenças sob o eixo do RDC/DTM de diagnóstico físico, nenhuma abordagem de tratamento universal foi determinada. Embora a fisiopatologia da dor miofascial permaneça incerta, uma das teorias aceitas é que a dor miofascial origina dos pontos-gatilho, que provocam um padrão de dor referida. Muitas modalidades de tratamento têm sido defendidas para controlar, estabilizar ou reverter essa hiperatividade muscular, entre elas, o uso de BTX-A, que vem apresentando resultados positivos. Alguns ensaios clínicos, no entanto, não conseguiram encontrar um benefício de injeções de BTX-A para pacientes com dor miofascial. Entre os problemas com os estudos atuais, estão a diversidade metodológica em projetos experimentais, amostras pequenas, e variabilidade dos locais de injeção. A BTX-A pode não ser uma terapia de primeira escolha para a dor miofascial, mas é uma alternativa útil ou tratamento adjuvante se medidas conservadoras falharem em produzir resultados satisfatórios ${ }^{21}$.

A BTX-A tem sido usada clinicamente para tratar uma variedade de condições, incluindo bruxismo e dor miofascial. Apesar de sua ampla aplicação, não há muita comprovação quanto a sua eficácia, pois a literatura consiste principalmente de estudos não controlados e abertos em vez de ensaios clínicos randomizados, duplo-cegos. O objetivo da pesquisa foi apresentar os argumentos contra o uso de BTX-A na gestão da dor miofascial, envolvendo os músculos da mastigação. Dentre os argumentos, o autor relata que o mecanismo de ação da BTX-A não aborda a etiologia do problema, apenas os sintomas, com isso o uso terapêutico da BTX-A baseia-se em várias teorias não provadas; não há estudos prospectivos, duplo-cego, randomizados, controlados por placebo suficientes para provar a eficácia da BTX-A para essa finalidade; o tratamento com BTX-A não é permanente, sendo necessário o retratamento, sendo assim uma opção cara. Com isso, o autor acredita que o tratamento com BTX-A apresenta maiores riscos aos pacientes comparado as formas convencionais, que são igualmente eficazes ${ }^{22}$.

Uma análise prospectiva de resultados foi realizada após a injeção de toxina botulínica em pacientes para controlar a dor miofascial mastigatória que não resolveram após as medidas conservadoras e que não aceitaram tomar doses baixas de relaxante muscular. Sessenta e dois pacientes foram avaliados pela EVA para dor no lado afetado antes do inicio do tratamento com BTX-A, após 6 semanas da injeção (50 unidades BTX-A em até 3 pontos, podendo ser nos músculos masseter, temporal, ou pterigóideo), e na abertura da boca medida em milímetros. Dos 62 pacientes tratados, 79\% apresentaram melhora na dor em mais de $25 \%$ na escala EVA. Dos 84 lados tratados, houve mais de $90 \%$ de resolução da dor em 25 dos lados, sendo que apenas 22 pacientes tiveram mais do que um local de injeção para o mesmo lado. A abertura bucal aumentou em média 0,9mm depois do tratamento. $\mathrm{O}$ tratamento diminuiu significativamente a dor dos pacientes em $57 \%$ em média 
e a duração da BTX-A foi de 3 a 5 meses. De acordo com os autores, a injeção de BTX-A não garante a resolução completa da dor miofascial, mas, geralmente, tem algum efeito benéfico na melhoria dos sintomas, e deve ser considerado um tratamento alternativo se métodos conservadores falharem ${ }^{23}$.

Após levantamento da literatura foi observado que a síndrome dolorosa miofascial (MPS) é a condição de dor crônica mais comum e é caracterizada por pontos-gatilho. Apesar de haver tratamentos atuais como fisioterapia, analgésicos, antidepressivos e injeções no ponto gatilho, a dor miofascial continua sendo uma condição de dor crônica desafiadora à prática clínica. Oito estudos duplo-cegos, randomizados e controlados foram selecionados para avaliar a eficácia de injeções de BTX-A nos pontos-gatilho ativos como um tratamento para redução da MPS. Os autores observaram que existem ensaios clínicos que suportam a eficácia de injeções de BTX-A nos pontos-gatilhos para MPS. No entanto, mais estudos clínicos randomizados, duplo cegos, multicêntricos e controlados devem ser feitos para fornecer prova conclusiva da eficácia da BTX-A no tratamento da dor miofascial ${ }^{24}$.

Com o objetivo de avaliar a eficácia da terapia com BTX-A para DTM, uma revisão sistemática foi realizada com em base em artigos relevantes publicados desde o inicio das pesquisas até outubro de 2014. Estudos selecionados foram publicações revisadas por pares de ensaios clínicos randomizados comparando BTX-A com qualquer intervenção alternativa ou placebo. Foram identificados cinco ensaios relevantes do estudo, envolvendo 117 participantes. Dois ensaios revelaram uma significativa diferença na redução da dor miofascial entre os grupos de BTX-A e placebo, outro ensaio mostrou a eficácia no alívio da dor em DTM tanto com o uso da BTX-A como na manipulação facial, enquanto os estudos não mostraram nenhuma diferença significativa entre os grupos placebo e BTX-A. Não foi possível realizar uma meta-análise devido a variações consideráveis nos métodos de estudo e avaliação dos resultados. Não foi possível alcançar um consenso sobre os benefícios terapêuticos da BTX no tratamento da DTM, sendo necessário que um desenho mais rigoroso dos ensaios seja realizado em estudos futuros ${ }^{25}$.

\section{DISCUSS ÃO}

A toxina botulínica tipo A foi utilizada nas DTM musculares ${ }^{14}$, incluindo dores miofasciais ${ }^{2,10,12,15,18-19,23}$ relacionadas a dores de cabeça ${ }^{20}$, desarranjos articulares internos, artralgia ou à combinação desses fatores ${ }^{1,13}$. A toxina também pode ser utilizada em indivíduos com dor miofascial e bruxismo ${ }^{19}$.

Foi observado na literatura, dentre os trabalhos avaliados, que as dosagens de BTX-A podem variar de $10 \mathrm{U}$ a $400 \mathrm{U}^{18}$, podendo ser feitas em sessão única ou em duas aplicações. Em geral, pode ser aplicada em até três pontos, preferencialmente, nos músculos elevadores da mandíbula, sendo os músculos masseter e temporal os mais escolhidos. Outra aplicação bastante comum é nos pontos gatilho, e há também relatos de aplicação nos músculos pterigoide lateral, pterigoide medial, platisma e digastrico.

Sabe-se que o ideal para avaliar o efeito de um tratamento é o acompanhamento longitudinal desde o pré-tratamento. Porém, há relatos de avaliação apenas pré-aplicação da BTX-A ou pós-aplicação imediata ou após 12 meses da aplicação de BTX-A e, na grande maioria dos estudos, avaliou-se apenas o pós-tratamento, que varia de 8 a 12 semanas. 
Poucos autores citaram o tempo de duração da toxina botulínica, podendo ser de 3 a 4 semanas, de 4 a 6 semanas ou de 3 a 5 meses $^{12,18,23}$.

Para alguns autores o tratamento com toxina botulinica tipo A é eficaz para o tratamento de pacientes com DTM ${ }^{1,12-13,15,18-19,24}$. Porem, alguns afirmam que, há eficácia no tratamento com toxina botulínica, mas apenas como uma segunda opção de tratamento se os métodos convencionais, como placas, não funcionarem ${ }^{20-21,23}$. Há também alguns autores que observaram boa atuação da toxina botulínica tipo A, porém, sugerem que evidencia cientifica mais consistente, por meio de estudos clínicos controlados e randomizados, são necessárias para comprovar os reais efeitos desse tratamento ${ }^{10-11,14,24}$.

Entretanto, alguns autores não acreditam na eficácia da toxina botulinica para o tratamento de pacientes com DTM, pois as pesquisas realizadas não mostraram resultados eficazes, sendo necessários mais estudos de longo prazo ${ }^{2,16-17,22}$, sendo que um estudo foi realizado para mostrar argumentos contra o uso de toxina botulínica, pois o autor acredita que o tratamento com BTX-A apresenta maiores riscos aos pacientes comparado as formas mais convencionais ${ }^{22}$.

\section{CONCLUSÃO}

A maioria dos estudos observou a eficácia da BTX-A na diminuição da dor de indivíduos com DTM. Porem é necessário que mais estudos clínicos randomizados, duplo cegos, multicêntricos e controlados sejam realizados para que a eficácia da BTX-A seja comprovada e para que um protocolo de atendimento seja realizado.

\section{REFERÊNCIAS}

1. Freund B, Schwartz M, Symington JM. Botulinum toxin: new treatment for temporomandibular disorders. Br J Oral Maxillofac Surg 2000; 5(38):466-71.

2. Ernberg M, Hedenberg-magnusson B, List T, Svensson P. Efficacy of botulinum toxin type A for treatment of persistent myofascial TMD pain: A randomized, controlled, double-blind multicenter study. Pain 2011; 9(152):1988-96.

3. Dworkin SF. Orofacial Pain: Prospective Evaluation and Risk Assessment (Oppera). J of Pain 2011; 3(11):T1-T3.

4. Dao TT, Lavigne GJ, Charbonneau A, Feine JS, Lund JP. The efficacy of oral splints in the treatment of myofascial pain of the jaw muscles: a controlled clinical trial. Pain 1994; 1(56): 85-94.

5. Turp JC, kowalski CJ, Stohler CS. Treatment-seeking patterns of facial pain patients: many possibilities, limited satisfaction. J of Orofac Pain 1998; 1(12): 61-6.

6. Fischer MJ, Reiners A, Kohnen R, Bernateck M, Gutenbrunner C, Fink M. et al. Do occlusal splints have an effect on complex regional pain syndrome? A randomized, controlled proof-of-concept trial. Clin J Pain 2008; 9(24): 776-83.

7. Tecco S, Teté S, Crincoli V, Festa MA, Festa F. Fixed orthodontic therapy in temporomandibular disorder (TMD) treatment: an alternative to intraoral splint. Cranio 2010; 1(28):30-42.

8 Niemela K, Korpela M, Raustia A, Ylostalo P, Sipila K. Efficacy of stabilisation splint treatment on temporomandibular disorders. J Oral Rehabil 2012; 11(39):799-804.

9. Dressler D, Saberi FA, Barbosa, ER. Botulinum toxin: Mechanisms of action. Arquivos de neuro-psiquiatria 2005; 1(63):180-85.

10. Guarda-nardini, Stecco LA, Stecco C, Masiero S, Manfredini D. Myofascial pain of the jaw muscles: Comparison of short-term effectiveness of botulinum toxin injections and fascial manipulation technique. Cranio 2012; 2(30):95-102.

11. Schwartz M, Freund B. Treatment of temporomandibular disorders with botulinum toxin. Clin J Pain 2002; 6(18):198-203.

12. Cheshire WP, Abashian SW, Mann JD. Botulinurn toxin in the treatment of myofascial pain syndrome. Pain 
1994; 1(59):65-9.

13. Freund B, Schwartz M, Symington JM. The use of botulinum toxin for the treatment of temporomandibular disorders: Preliminary findings. J Oral Maxillofac Surg 1999; 8(57):916-20.

14. Lindern JJ. Type A botulinum toxin in the treatment of chronic facial pain associated with temporomandibular dysfunction. Acta Neurol Belg2001;1(101):39-41.

15. Lindern JJ, Niederhagen B, Berge S, Appel T. Type A botulinum toxin in the treatment of chronic facial pain associated with masticatory hyperactivity. J Oral Maxillofac Surg 2003; 7(61):774-78.

16. Ho KY, Tan KH. Botulinum toxin A for myofascial trigger point injection: A qualitative systematic review. Eur J Pain 2006; 5(11):529-27.

17. Qerama E, Fuglsang-frederiksen A, Bach FW, Jensen TS. A double-blind, controlled study of botulinum toxin A in chronic myofascial pain. Neurology 2006; 2(67):241-45.

18. Gobel H, Heinze A, Reichel G, Hefter H, Benecke R. Efficacy and safety of a single botulinum type A toxin complex treatment (BTX-A) for the relief of upper back myofascial pain syndrome: Results from a randomized double-blind placebo-controlled multicentre study. Pain 2006; 1-2(125):82-8.

19. Guarda-nardini 1, Manfredini D, Salamone M, Salmaso L, Tonello S, Ferronato G. Efficacy of botulinum toxin in treating myofascial pain in bruxers: A controlled placebo pilot study. Cranio 2008; 2(26):126-35.

20. Venancio RA, Alencar FGP, Zamperini C. Botulinum toxin, lidocaine, and dry-needling injections in patients with myofascial pain and headaches. Cranio 2009; 1(27):46-56.

21. Fallah HM, Currimbhoy S. Use of botulinum toxin A for treatment of myofascial pain and dysfunction. J Oral Maxillofac Surg 2012; 5(70):1243-45.

22. Laskin DM. Botulinum toxin A in the treatment of myofascial pain and dysfunction: The case against its use. J Oral Maxillofac Surg 2012; 5(70):1240-42.

23. Sidebottom AJ, Patel AA, Amin J. Botulinum injection for the management of myofascial pain in the masticatory muscles. A prospective outcome study. British J Oral Maxillofac Surg 2013; 3(51):199-205.

24. Zhou JY, Wang D. An update on botulinum toxin A injections of trigger points for myofascial pain. Curr Pain Headache Rep 2014; 1(18):386.

25. Chen YW, Chiu YW, Chen CY, Chang SK. Botulinum toxin therapy for temporomandibular joint disorders: a systematic review of randomized controlled trials. Int J Oral Maxillofac Surg 2015; 8(44):1018-26. 


\begin{tabular}{|c|c|c|c|}
\hline & Metodologia & Resultado & Conclusão \\
\hline $\begin{array}{l}\text { Cheshire, } \\
\text { Abashian e Mann } \\
\text { (1994) }\end{array}$ & $\begin{array}{l}6 \text { pacientes com síndrome dolorosa } \\
\text { miofascial receberam duas aplicações } \\
\text { de } 50 U \text { de BTX-A ou } 4 \mathrm{ml} \text { de solução } \\
\text { salina nos pontos gatilhos com inter- } \\
\text { valos semanais durante } 4 \text { semanas } \\
\text { após cada injeção e } 8 \text { semanas após a } \\
\text { injeção final }\end{array}$ & $\begin{array}{l}\text { Quatro pacientes apresentaram } \\
\text { redução da dor de pelo menos } \\
30 \% \text {, após aplicação de BTX-A. Os } \\
\text { pacientes relataram que a melhora } \\
\text { após a ultima injeção durou de } 3 \text { a } \\
4 \text { semanas }\end{array}$ & $\begin{array}{l}\text { O tratamento com a BTX-A mostrou-se } \\
\text { eficaz para, mas os resultados devem } \\
\text { ser confirmados por um estudo mais } \\
\text { amplo. }\end{array}$ \\
\hline $\begin{array}{l}\text { Freund, Schwartz } \\
\text { e Symington } \\
(1999)\end{array}$ & $\begin{array}{l}15 \text { pacientes com DTM receberam } \\
150 \mathrm{U} \text { de BTX-A Músculos masseter } \\
\text { (50U em cada) e temporal (25U em } \\
\text { cada) sendo avaliados com intervalos } \\
\text { de } 2 \text { semanas, durante } 8 \text { semanas }\end{array}$ & $\begin{array}{l}\text { Todas as medidas de resultados, } \\
\text { com exceção da força de mordida, } \\
\text { mostraram uma diferença signifi- } \\
\text { cativa. }\end{array}$ & $\begin{array}{l}\text { Em quatro dos cinco resultados medi- } \\
\text { dos apresentaram melhoras estatisti- } \\
\text { camente significativas após as injeções } \\
\text { de BTX-A }\end{array}$ \\
\hline $\begin{array}{l}\text { Freund, Schwartz } \\
\text { e Symington } \\
(2000)\end{array}$ & $\begin{array}{l}46 \text { pacientes com DTM miofascial } \\
\text { (com desarranjos articulares internos } \\
\text { ou artralgia, ou a combinação dos } \\
\text { dois) receberam 150U de BTX-A nos } \\
\text { músculos masseter e temporal com } \\
\text { maior massa muscular à palpação, e } \\
\text { maior atividade EMG e foram ava- } \\
\text { liados a cada duas semanas, durante } \\
\text { oito semanas. }\end{array}$ & $\begin{array}{l}\text { Os resultados entre a avalia- } \\
\text { ção pré-tratamento e as quatro } \\
\text { avaliações de acompanhamento } \\
\text { demonstraram diferenças signifi- } \\
\text { cativas, produzindo melhoras na } \\
\text { dor, na função, na abertura de boca } \\
\text { e na sensibilidade a palpação em } \\
\text { pacientes com DTM }\end{array}$ & $\begin{array}{l}\text { Embora o estudo não tenha sido } \\
\text { controlado, os autores obtiveram } \\
\text { resultados significativos nas injeções } \\
\text { de BTX-A }\end{array}$ \\
\hline Lindern (2001) & $\begin{array}{l}40 \text { pacientes com DTM muscular rece- } \\
\text { beram 200U de BTX-A nos músculos } \\
\text { masseter, temporal e platisma. Em } 8 \\
\text { casos, foi administrada nos músculos } \\
\text { pterigoide lateral, digastrico e pteri- } \\
\text { goideo medial. }\end{array}$ & $\begin{array}{l}\text { Os resultados mostraram que } 80 \% \\
\text { dos pacientes obtiveram melhoras } \\
\text { por uma redução média de } 45 \% \\
\text { sobre uma escala de dor análoga } \\
\text { visual. Foi observado melhora } \\
\text { durante } 3 \text { a } 12 \text { meses. }\end{array}$ & $\begin{array}{l}\text { Embora os resultados sejam encoraja- } \\
\text { dores, é necessário serem confirmados } \\
\text { por estudos randomizados controlados. }\end{array}$ \\
\hline $\begin{array}{l}\text { Schwartz e } \\
\text { Freund (2002) }\end{array}$ & $\begin{array}{l}\text { Foi realizada uma pesquisa para } \\
\text { descrever os tipos mais prevalentes } \\
\text { de DTM e o tratamento com a BTX-A, } \\
\text { a dosagem e os locais das injeções de } \\
\text { cada músculo. }\end{array}$ & $\begin{array}{l}\text { No músculo temporal: } 5 \text { injeções } \\
\text { de } 5 \text { a } 25 \mathrm{U} \text { BTX-A; músculo masse- } \\
\text { ter: } 5 \text { injeções de } 25 \text { a } 50 \mathrm{U} \text { BTX-A; } \\
\text { músculos pterigoide medial: } 2 \text { a } 3 \\
\text { injeções de } 5 \text { a } 25 \mathrm{U} \text { de BTX-A; mús- } \\
\text { culo pterigoide lateral: } 1 \text { injeção } \\
\text { de } 5 \text { a } 10 \mathrm{U} \text { BTX-A com orientação } \\
\text { EMG. }\end{array}$ & $\begin{array}{l}\text { Os autores afirmam que a toxina } \\
\text { botulínica está emergindo como uma } \\
\text { ferramenta clínica muito potente e } \\
\text { valiosa para o cuidado de diagnóstico } \\
\text { e de terapêutica de DTM, mas ensaios } \\
\text { clínicos são necessários para compro- } \\
\text { var sua eficácia. }\end{array}$ \\
\hline $\begin{array}{l}\text { Lindern et al. } \\
\text { (2003) }\end{array}$ & $\begin{array}{l}90 \text { pacientes com dor miofacial crôni- } \\
\text { ca, sendo } 30 \text { pacientes que receberam } \\
\text { tratamento placebo e os demais } 35 \mathrm{U} \\
\text { de BTX-A nos músculo masseter, } \\
\text { temporal e pterigoide medial }\end{array}$ & $\begin{array}{l}91 \% \text { dos pacientes que receberam } \\
\text { toxina botulínica demonstraram } \\
\text { melhora de aproximadamente } 3.2 \\
\text { em media na escala VAS }\end{array}$ & $\begin{array}{l}\text { Os autores concluíram que a injeção } \\
\text { local de BTX-A é um método inovador } \\
\text { e eficiente para o tratamento da dor } \\
\text { facial crônica associada com hiperati- } \\
\text { vidade dos músculos mastigatórios }\end{array}$ \\
\hline Ho e Tan (2006) & $\begin{array}{l}\text { Revisão sistemática para avaliar a efi- } \\
\text { cácia da BTX-A em comparação com } \\
\text { outros tratamentos (anestésico local, } \\
\text { corticosteroides, injeção de solução } \\
\text { salina e agulhamento seco) e placebo. }\end{array}$ & $\begin{array}{l}\text { Um estudo concluiu que BTX-A } \\
\text { foi eficaz, e quatro concluíram que } \\
\text { não era eficaz para reduzir a dor } \\
\text { decorrente de pontos-gatilho. }\end{array}$ & $\begin{array}{l}\text { Os autores não apoiaram a utilização } \\
\text { de BTX-A na redução da dor asso- } \\
\text { ciada com os pontos-gatilho, pois as } \\
\text { evidências não suportam sua eficácia e } \\
\text { os dados são limitados e clinicamente } \\
\text { heterogêneos. }\end{array}$ \\
\hline $\begin{array}{l}\text { Qerama et al. } \\
(2006)\end{array}$ & $\begin{array}{l}30 \text { pacientes com dor miofacial } \\
\text { receberam } 50 \mathrm{U} \text { de BTX-A e } 0,25 \mathrm{~mL} \\
\text { de solução salina isotônica nos pontos } \\
\text { gatilho. Foram avaliados após } 3 \text { e } 28 \\
\text { dias da aplicação. }\end{array}$ & $\begin{array}{l}\text { Houve redução significativa na } \\
\text { atividade da placa motora e no } \\
\text { padrão de interferência no EMG } \\
\text { após o uso do BTX-A, mas não } \\
\text { teve efeito sobre qualquer dor ou o } \\
\text { limiar da dor em comparação com } \\
\text { solução salina isotônica. }\end{array}$ & $\begin{array}{l}\text { Os autores concluíram que os resulta- } \\
\text { dos não suportam um efeito antino- } \\
\text { ciceptivo e analgésico específico de } \\
\text { BTX-A e que mais estudos precisam } \\
\text { ser realizados. }\end{array}$ \\
\hline
\end{tabular}




\begin{tabular}{|c|c|c|c|}
\hline $\begin{array}{l}\text { Gobel et al. } \\
\text { (2006) }\end{array}$ & $\begin{array}{l}145 \text { pacientes com síndrome dolorosa } \\
\text { miofascial receberam } 40 \mathrm{U} \text { de injeções } \\
\text { de BTX-A }(\mathrm{n}=75) \text { ou injeções de solu- } \\
\text { ção salina }(\mathrm{n}=70) \text { nos } 10 \text { pontos gatilho } \\
\text { mais sensíveis e foram avaliados } \\
\text { durante } 12 \text { semanas }\end{array}$ & $\begin{array}{l}\text { Em comparação com placebo, o } \\
\text { grupo que recebeu injeções de } \\
\text { BTX-A apresentou diminuição da } \\
\text { intensidade da dor significativa- } \\
\text { mente maior durante as semanas } \\
\text { 5-8, e mais dias por semana sem } \\
\text { dor entre } 5 \text { e } 12 \text { semanas, com } \\
\text { duração de } 4 \text { a } 6 \text { semanas. }\end{array}$ & $\begin{array}{l}\text { Os autores concluíram que em pacien- } \\
\text { tes com síndrome dolorosa miofascial } \\
\text { na parte superior das costas, que rece- } \\
\text { beram injeções de BTX-A melhoraram } \\
\text { significativamente os níveis de dor }\end{array}$ \\
\hline $\begin{array}{l}\text { Guarda-Nardini } \\
\text { et al. (2008) }\end{array}$ & $\begin{array}{l}20 \text { pacientes com dor miofascial } \\
\text { associado com bruxismo receberam } \\
\text { injeções num total de 100U de BTX-A, } \\
\text { sendo } 30 \mathrm{U} \text { em cada músculo masseter } \\
\text { e } 20 \mathrm{U} \text { em cada músculo temporal } \\
\text { anterior (G1) em uma sessão única e } \\
\text { um grupo controle recebeu injeções } \\
\text { de placebo salino (G2). }\end{array}$ & $\begin{array}{l}\text { O estudo mostrou melhora objetiva } \\
\text { (amplitude dos movimentos man- } \\
\text { dibulares) e subjetiva (dor em re- } \\
\text { pouso e dor durante a mastigação) } \\
\text { havendo uma melhora significati- } \\
\text { vamente maior nos resultados do } \\
\text { grupo tratado com BTX-A do que } \\
\text { no grupo tratado com placebo. }\end{array}$ & $\begin{array}{l}\text { apoiaram a eficácia da BTX-A para } \\
\text { reduzir os sintomas de dor miofascial } \\
\text { em indivíduos com bruxismo e for- } \\
\text { neceram dado piloto que precisa ser } \\
\text { confirmados por novas pesquisas com } \\
\text { amostras maiores. }\end{array}$ \\
\hline $\begin{array}{l}\text { Venancio, Alen- } \\
\text { car e Zamperini } \\
(2009)\end{array}$ & $\begin{array}{l}45 \text { pacientes com dor miofascial e } \\
\text { dores de cabeça foram divididos em } \\
\text { três grupos: G1 com agulhas secas, G2 } \\
\text { com } 0,25 \% \text { de lidocaína a } 0,25 \% \text { e G3 } \\
\text { com } 25 \text { a } 50 \mathrm{U} \text { de BTX que receberam } \\
\text { injeções nos pontos gatilho. }\end{array}$ & $\begin{array}{l}\text { Todos os grupos apresentaram } \\
\text { resultados estatisticamente favorá- } \\
\text { veis para os requisitos avaliados. }\end{array}$ & $\begin{array}{l}\text { A BTX deveria ser reservada para } \\
\text { casos refratários, em que não podem } \\
\text { ser atingidos os efeitos esperados }\end{array}$ \\
\hline $\begin{array}{l}\text { Ernberg et al. } \\
\text { (2011) }\end{array}$ & $\begin{array}{l}21 \text { pacientes com dor miofascial } \\
\text { crônica receberam tanto } 50 \mathrm{U} \text { de } \\
\text { BTX-A quanto uma solução salina } \\
\text { isotônica nos músculos masseter com } \\
\text { sintomatologia dolorosa. }\end{array}$ & $\begin{array}{l}\text { Não houve diferença estatistica- } \\
\text { mente significante na redução } \\
\text { da dor com toxina botulínica e } \\
\text { solução salina, porém a toxina foi } \\
\text { mais rápida. }\end{array}$ & $\begin{array}{l}\text { A BTX-A não é eficaz como um } \\
\text { complemento ao tratamento conserva- } \\
\text { dor em pacientes com dor miofascial } \\
\text { persistente }\end{array}$ \\
\hline $\begin{array}{l}\text { Guarda-Nardini } \\
\text { et al. (2012) }\end{array}$ & $\begin{array}{l}30 \text { pacientes com dor miofascial foram } \\
\text { divididos em dois grupos: grupo A } \\
\text { recebeu injeções de } 150 \text { U de BTX-A } \\
\text { em uma sessão única e o Grupo B } 3 \\
\text { sessões de } 50 \text { minutos (uma por se- } \\
\text { mana) de manipulação facial, ambos } \\
\text { nos músculos masseter e temporal. } \\
\text { Foram avaliados no início, no final do } \\
\text { tratamento e em um período de três } \\
\text { meses após o tratamento. }\end{array}$ & $\begin{array}{l}\text { Os dois tratamentos demonstraram } \\
\text { quase igualmente eficazes, sendo } \\
\text { a manipulação facial ligeiramente } \\
\text { superior na redução da percep- } \\
\text { ção da dor subjetiva (VAS), e as } \\
\text { injeções de BTX-A sendo superior } \\
\text { no aumento da amplitude de mo- } \\
\text { vimento da mandíbula }\end{array}$ & $\begin{array}{l}\text { Estudos futuros com amostras maiores } \\
\text { e acompanhamento longitudinal por } \\
\text { maior tempo são necessários a fim de } \\
\text { elucidar as melhores estratégias de } \\
\text { tratamento para este quadro clinico. }\end{array}$ \\
\hline $\begin{array}{l}\text { Fallah e Currim- } \\
\text { bhoy (2012) }\end{array}$ & $\begin{array}{l}\text { Revisao de literatura para determi- } \\
\text { nar uma abordagem de tratamento } \\
\text { universal para os distúrbios da dor } \\
\text { miofascial. }\end{array}$ & $\begin{array}{l}\text { Alguns ensaios clínicos não conse- } \\
\text { guiram encontrar um benefício de } \\
\text { injeções de BTX-A para pacientes } \\
\text { com dor miofascial. }\end{array}$ & $\begin{array}{l}\text { A BTX-A pode não ser uma tera- } \\
\text { pia de primeira escolha para a dor } \\
\text { miofascial, mas é uma alternativa útil } \\
\text { ou tratamento adjuvante se medidas } \\
\text { conservadoras falharem em produzir } \\
\text { resultados satisfatórios. }\end{array}$ \\
\hline Laskin (2012) & $\begin{array}{l}\text { O objetivo da pesquisa foi apresentar } \\
\text { os argumentos contra o uso de BTX-A } \\
\text { na gestão da dor miofascial, envolven- } \\
\text { do os músculos da mastigação. }\end{array}$ & $\begin{array}{l}\text { O mecanismo de ação da BTX-A } \\
\text { não aborda a etiologia do proble- } \\
\text { ma; não há estudos prospectivos, } \\
\text { duplo-cego, randomizados, contro- } \\
\text { lados por placebo suficientes para } \\
\text { provar a eficácia da BTX-A para } \\
\text { essa finalidade; o tratamento com } \\
\text { BTX-A não é permanente, sendo } \\
\text { necessário o retratamento. }\end{array}$ & $\begin{array}{l}\text { O autor acreditam que o tratamento } \\
\text { com BTX-A apresentam maiores } \\
\text { riscos aos pacientes comparado as } \\
\text { formas mais convencionais, que são } \\
\text { igualmente eficazes no controle da do } \\
\text { miofacial. }\end{array}$ \\
\hline
\end{tabular}




\begin{tabular}{|c|c|c|c|}
\hline $\begin{array}{l}\text { Sidebottom, Patel } \\
\text { e Aminb (2012) }\end{array}$ & $\begin{array}{l}62 \text { pacientes com dor miofascial mas- } \\
\text { tigatória receberam } 50 \mathrm{U} \text { BTX-A em } \\
\text { até } 3 \text { pontos nos músculos masseter, } \\
\text { temporal, ou pterigoide. Foram ava- } \\
\text { liados antes do inicio do tratamento e } \\
\text { após } 6 \text { semanas }\end{array}$ & $\begin{array}{l}\text { O tratamento diminuiu significa- } \\
\text { tivamente a dor dos pacientes em } \\
57 \% \text { em média }\end{array}$ & $\begin{array}{l}\text { A injeção de BTX-A não garante a } \\
\text { resolução completa da dor miofascial } \\
\text { mas, geralmente, tem algum efeito } \\
\text { benéfico na melhoria dos sintomas e } \\
\text { deve ser considerado um tratamento } \\
\text { alternativo se métodos conservadores } \\
\text { falharem. }\end{array}$ \\
\hline $\begin{array}{l}\text { Zhou e Wang } \\
\text { (2014) }\end{array}$ & $\begin{array}{l}\text { Oito estudos duplo-cegos, randomiza- } \\
\text { dos e controlados foram selecionados } \\
\text { para avaliar a eficácia de injeções } \\
\text { de BTX-A nos pontos-gatilho ativos } \\
\text { como um tratamento para redução da } \\
\text { dor miofacial. }\end{array}$ & $\begin{array}{l}\text { Os autores observaram que exis- } \\
\text { tem ensaios clínicos que suportam } \\
\text { a eficácia de injeções de BTX-A nos } \\
\text { pontos-gatilhos para dor miofacial }\end{array}$ & $\begin{array}{l}\text { Mais estudos clínicos com objetivo de } \\
\text { minimizar efeito placebo, doses repe- } \\
\text { tidas e cobertura adequada de pontos- } \\
\text {-gatilho deve ser feita para fornecer } \\
\text { provas conclusivas da eficácia da BTX- } \\
\text {-A no tratamento da dor miofascial. }\end{array}$ \\
\hline Chen et al. (2015) & $\begin{array}{l}\text { Uma revisão sistemática foi realizada } \\
\text { com em base em artigos relevantes } \\
\text { publicados desde o inicio das pesqui- } \\
\text { sas até outubro de 2014, sendo selecio- } \\
\text { nadas publicações revisadas por pares } \\
\text { de ensaios clínicos randomizados } \\
\text { comparando BTX-A com qualquer } \\
\text { intervenção alternativa ou placebo. }\end{array}$ & $\begin{array}{l}\text { Dois ensaios revelaram diferença } \\
\text { na redução da dor miofascial entre } \\
\text { os grupos de BTX-A e placebo, } \\
\text { outro ensaio mostrou a eficácia no } \\
\text { alívio da dor em DTM tanto com } \\
\text { o uso da BTX-A como na manipu- } \\
\text { lação facial, enquanto os estudos } \\
\text { não mostraram nenhuma diferença } \\
\text { significativa entre os grupos place- } \\
\text { bo e BTX-A. }\end{array}$ & $\begin{array}{l}\text { Os autores não alcançaram um con- } \\
\text { senso sobre os benefícios terapêuticos } \\
\text { da BTX no tratamento da DTM, sendo } \\
\text { assim necessário que um desenho } \\
\text { mais rigoroso dos ensaios seja realiza- } \\
\text { do em estudos futuros. }\end{array}$ \\
\hline
\end{tabular}

